\title{
MULTI-VALUED MONOTONE NONLINEAR MAPPINGS AND DUALITY MAPPINGS IN BANACH SPACES
}

\author{
BY \\ FELIX E. BROWDER(1)
}

Introduction. Let $X$ be a reflexive real Banach space, $X^{*}$ its conjugate space, $(w, u)$ the pairing between $w$ in $X^{*}$ and $u$ in $X$. We consider multi-valued mappings $T$ of $X$ into $X^{*}$ (i.e, mappings in the ordinary sense of $X$ into $2^{X^{*}}$ ) which are monotone, i.e., if $v \in T(u), v_{1} \in T\left(u_{1}\right)$ for $u$ and $u_{1}$ in $X$, then

$$
\left(v-v_{1}, u-u_{1}\right) \geqq 0 .
$$

It is our object in the present paper to generalize to the multi-valued case the results obtained in a number of recent papers by the author and G. J. Minty for single-valued mappings $T$ (cf. [2]-[14]). The first results for multi-valued mappings for $X$ a Hilbert space have been obtained in an unpublished paper of Minty [15]. The methods of [15] are not directly extendable to more general spaces, but our discussion of the finite-dimensional case (Lemma 2.1) has been very much influenced by the manuscript of [15] which Minty has recently transmitted to the author. (The basic result of [15] is stated at the end of $\$ 2$ below.)

Our results for general multi-valued monotone mappings have an interesting specific application given in $\S 3$ below to the generalization of a theorem of Beurling and Livingston [1] on duality mappings in Banach spaces. In a previous paper [12], we showed that for strictly convex reflexive spaces, this theorem could be obtained from results on single-valued monotone mappings. In $\S 3$ below we give a generalization of this theorem to general reflexive Banach spaces which runs as follows: Let $X$ be a reflexive Banach space, $\phi(r)$ a non-negative nondecreasing function on $R^{1}$ with $\phi(0)=0$. The duality map $T$ of $X$ with respect to $\phi$ is defined by

$$
T(u)=\left\{\begin{array}{l}
v \mid v \in X^{*},\|v\|=\phi(\|u\|) \\
(v, u)=\|v\| \cdot\|u\|
\end{array}\right.
$$

Received by the editors February 21, 1964.

(1) The preparation of this paper was partially supported by NSF grants 19751 and GP-2283 the Sloan Foundation, and the Army Research Office (Durham) through grant DA-ARO(D)31-124-G455. 
Let $Y$ be a closed subspace of $X, Y^{\perp}$ its annihilator in $X^{*}, v_{0}$ and $w_{0}$ arbitrary elements of $X$ and $X^{*}$, respectively. Then

is nonempty.

$$
T\left(Y+v_{0}\right) \cap\left(Y^{\perp}+w_{0}\right)
$$

$\S 1$ is devoted to the study of maximal monotonic mappings and of a very weak continuity property for multi-valued mappings which we have called vague continuity and which plays a key role in our discussion. $\$ 2$ contains the proof of the basic results on general multi-valued monotonic mappings. $\S 3$ contains the discussion of duality mappings.

1. Let $X$ be a reflexive Banach space over the reals, $X^{*}$ its conjugate space. We denote the pairing between $w$ in $X^{*}$ and $u$ in $X$ by $(w, u)$. We denote by $X \times X^{*}$ the product space of $X$ and $X^{*}$ whose elements will be written as $[u, w]$ and with norm

$$
\|[u, w]\|=\left\{\|u\|_{X}^{2}+\|w\|_{X^{*}}^{2}\right\}^{1 / 2} .
$$

We consider multi-valued mappings $T$ of $X$ into $X^{*}$, where $T$ assigns to each $u$ in $X$, a subset $T(u)$ (possibly empty) of $X^{*}$.

To make our discussion of multi-valued mappings more intuitive by tying the formalism of our arguments closer to the single-valued case, we introduce the following notational convention:

Convention. If $V$ is a subset of $X^{*}, u$ an element of $X$, then $(V, u)$ will denote the set $\{(v, u) \mid v \in V\}$. Similarly if $V$ and $W$ are subsets of $X^{*}$, then $(V-W, u)$ will denote the set $\{(v-w, u) \mid v \in V, w \in W\}$. If $c$ is a real number, and $R_{0}$ is $a$ set of real numbers, $R_{0} \geqq c$ (or $R_{0} \leqq c$ ) will denote the sets of inequalities $r \geqq c$ for $r \in R_{0}$ (or $r \leqq c$ for $r \in R_{0}$ ). If a set $V$ appears several times in a single equation or inequality, the equation or inequality is assumed to hold for each $v$ in $V$, with the same $v$ chosen at all points of occurrence of $V$ in the given equation or inequality.

Definition 1.1. Let $T$ be a (possibly) multi-valued map from $X$ to $X^{*}$. Then $T$ is said to be monotone if

$$
\left(T(u)-T\left(u_{1}\right), u-u_{1}\right) \geqq 0
$$

for all $u$ and $u_{1}$ in $X$.

Definition 1.2. The graph $G(T)$ is the subset of $X \times X^{*}$ given by

$$
G(T)=\{[u, w] \mid w \in T(u), u \in X\} .
$$

We say that $T \subseteq T_{1}$ if $G(T) \subseteq G\left(T_{1}\right)$.

DEFINITION 1.3. $T$ is said to be maximal monotone if $T$ is monotone and if for every monotone $T_{1}$ such that $T \subseteq T_{1}$, we have $T=T_{1}$.

If $S$ is a subset of $X$ or $X^{*}, K(S)$ will denote its convex closure, i.e., the smallest 
closed convex set containing $S$. $S$ is said to surround 0 if every ray $\{t w \mid t>0\}$ for $w \neq 0$ intersects $S$.

Lemma 1.1. Let $T$ be a maximal monotone multi-valued map from $X$ to $X^{*}$. Then:

(a): For every $u$ in $X, T(u)$ is a closed convex subset of $X^{*}$.

(b) If $\left\{u_{k}\right\}$ and $\left\{v_{k}\right\}$ are sequences in $X$ and $X^{*}$, respectively, such that $u_{k} \rightarrow u_{0}$ strongly in $X, v_{k} \in T\left(u_{k}\right)$, and $v_{k} \rightarrow v_{0}$ weakly in $X^{*}$, then $v_{0} \in T\left(u_{0}\right)$.

Proof of Lemma 1.1. Proof of (a). For $u, u_{1}$ in $X$ and $v, v_{0} \in T(u), v_{1} \in T\left(u_{1}\right)$, we have

$$
\begin{aligned}
& \left(v-v_{1}, u-u_{1}\right) \geqq 0, \\
& \left(v_{0}-v_{1}, u-u_{1}\right) \geqq 0 .
\end{aligned}
$$

If $0 \leqq t \leqq 1, v_{t}=t v+(1-t) v_{0}$, we have

$$
\left(v_{t}-v_{1}, u-u_{1}\right)=t\left(v-v_{1}, u-u_{1}\right)+(1-t)\left(v_{0}-v_{1}, u-u_{1}\right) \geqq 0 .
$$

If we add $v_{t}$ to $T(u)$ therefore to obtain a larger mapping $T_{1}$, it follows that $T_{1}$ is monotone. Since $T$ is maximal monotone, it follows that $v_{t} \in T(u)$, i.e., $T(u)$ is convex. Similarly $T(u)$ is closed.

Proof of (b). Let $u$ be any element of $X, v$ any element of $T(u)$. For every $k$, we have

$$
\left(v_{k}-v, u_{k}-u\right) \geqq 0 .
$$

Since $u_{k}-u$ converges strongly to $u_{0}-u$ while $v_{k}-v$ converges weakly to $v_{0}-v$, we have

$$
\left(v_{k}-v, u_{k}-u\right) \underset{k \rightarrow \infty}{\longrightarrow}\left(v_{0}-v, u_{0}-u\right)
$$

Hence

$$
\left(v_{0}-v, u_{0}-u\right) \geqq 0
$$

for every $u$ in $X, v \in T(u)$. By the maximal monotonicity of $T$, it follows that $v_{0} \in T(u)$. Q.E.D.

DEFINITION 1.4. If $T$ is a multi-valued transformation from $X$ to $X^{*}$, its domain $D(T)$ is defined to be the set of $u$ in $X$ for which $T(u) \neq \varnothing$.

Definition 1.5. If $T$ is a multi-valued mapping from $X$ to $X^{*}, T$ is said to be vaguely continuous if $D(T)$ is a dense linear subset of $X$ and the following condition is satisfied.

For each pair $u_{0}$ and $u_{1}$ of $D(T)$, there exists a sequence $\left\{t_{n}\right\}$ of positive real numbers with $t_{n} \rightarrow 0$ as $n \rightarrow+\infty$ and an element $v_{1}$ of $K\left(T\left(u_{0}\right)\right)$ such that if $u_{n}=t_{n} u_{1}+\left(1-t_{n}\right) u_{0}$, there exist elements $v_{n} \in K\left(T\left(u_{n}\right)\right)$ such that $v_{n} \rightarrow v_{1}$ weakly in $X^{*}$. 
If $T$ is a single-valued mapping, vague continuity of $T$ is a weakening of the condition of hemi-continuity of $T$ as introduced by the author in [5] (i.e., $T$ continuous from each segment in $D(T)$ to the weak topology of $X^{*}$ ),

THEOREM 1.1. Let $T$ be a maximal monotone mapping of $X$ into $X^{*}$ such that $D(T)$ is a dense linear subset of $X$ and for each closed line segment $S_{0}$ in $D(T)$, there is a bounded set $S_{1}$ in $X^{*}$ such that $T(u) \cap S_{1} \neq \varnothing$ for $u \in S_{0}$.

Then $T$ is vaguely continuous and $T(u)$ is a closed convex set for every $u$ in $D(T)$.

Proof of Theorem 1.1. We know from the maximal monotonicity of $T$ and part (a) of Lemma 1.1 that $T(u)$ is a closed convex set in $X^{*}$ for every $u$ in $D(T)$. It follows from the hypotheses of our theorem that $D(T)$ is a dense linear subset of $X$. We need only to show that the condition of Definition 1.5 is satisfied.

Let $S_{0}$ be the closed line segment $\left\{u_{t}=t u_{1}+(1-t) u_{0} \mid 0 \leqq t \leqq 1\right\}$ in $D(T)$. By hypothesis, there exists a constant $M$ depending on $S_{0}$ such that for each $u_{t}$ in $S$, we may find $v_{t}$ in $T\left(u_{t}\right)$ with $\left\|v_{t}\right\| \leqq M$. By the weak compactness of the closed ball in the reflexive Banach space $X^{*}$, we may choose a sequence $\left\{t_{n}\right\}$ with $t_{n}>0, t_{n} \rightarrow 0$ as $n \rightarrow+\infty$ and $v_{t_{n}} \rightarrow v_{1}$ weakly in $X^{*}$ as $n \rightarrow+\infty$. However, $u_{t_{n}} \rightarrow u_{0}$ strongly in $X$. Since $T$ is maximal monotone, it follows from Lemma $1.1(\mathrm{~b})$ that $v_{1} \in T\left(u_{0}\right)$. Q.E.D.

We have a converse for Theorem 1.1, namely:

THEOREM 1.2. Let $T$ be a multi-valued mapping of $X$ into $X^{*}$ for which all of the following conditions are satisfied.

(a) $T$ is monotone.

(b) $D(T)=X$ and $T(u)$ is a closed convex set for each $u$ in $X$.

(c) $T$ is vaguely continuous.

Then $T$ is maximal monotone.

Proof of Theorem 1.2. Suppose $T \subseteq T_{1}$, where $T_{1}$ is monotone and $v_{0} \in T_{1}\left(u_{0}\right)$. We must show that $v_{0} \in T\left(u_{0}\right)$. By the monotonicity of $T_{1}$, we know that for every $u$ in $X$ and $v \in T(u)$, we have

$$
\left(v-v_{0}, u-u_{0}\right) \geqq 0 .
$$

Suppose $v_{0}$ does not lie in $T\left(u_{0}\right)$. Since $T\left(u_{0}\right)$ is closed and convex there exists $w$ in $X$ such that

$$
\left(v_{0}, w\right)>\left(T\left(u_{0}\right), w\right) .
$$

For real $t>0$, set $u_{t}=u_{0}+t w$. For any $v$ in $T\left(u_{t}\right)$, we have

$$
t\left(v-v_{0}, w\right) \mid \geqq 0,
$$

i.e., 


$$
\left(v-v_{0}, w\right) \geqq 0, \quad v \in T\left(u_{t}\right),
$$

or

$$
\left(T\left(u_{t}\right)-v_{0}, w\right) \geqq 0
$$

Hence

$$
\left(T\left(u_{t}\right)-T\left(u_{0}\right), w\right) \geqq\left(v_{0}-T\left(u_{0}\right), w\right)
$$

for all $t>0$. Hence, choosing $\left\{v_{k}\right\}$ for the segment $\left\{u_{t}=u_{0}+t w \mid 0 \leqq t \leqq 1\right\}$ we have $v_{k} \in T\left(u_{k}\right)$, where $u_{k}=u_{0}+t_{k} w\left(t_{k} \rightarrow 0\right)$ with $v_{k} \rightarrow v_{1}$ weakly in $X^{*}$ for some $v_{1}$ in $T\left(u_{0}\right)$. Hence

which implies that

$$
\left(v_{k}-v_{1}, w\right) \geqq\left(v_{0}-v_{1}, w\right),
$$

$$
0 \geqq\left(v_{0}-v_{1}, w\right) \geqq\left(v_{0}-T\left(u_{0}\right), w\right)>0,
$$

yielding a contradiction. Q.E.D.

LEMMA 1.2. If $T$ is a maximal monotone multi-valued mapping from $X$ to $X^{*}$ and if for sequences $\left\{u_{k}\right\}$ and $\left\{v_{k}\right\}$ from $X$ and $X^{*}$, respectively, we have

and

$$
v_{k} \in T\left(u_{k}\right)
$$

$$
\begin{gathered}
u \rightarrow g_{0} \text { weakly in } X, \\
v_{k} \rightarrow v_{0} \text { strongly in } X^{*},
\end{gathered}
$$

then $v_{0} \in T\left(u_{0}\right)$.

Proof of Lemma 1.2. For $u$ in $X, v \in T(u)$, we have for every $k$

$$
\left(v_{k}-v, u_{k}-u\right) \geqq 0 \text {. }
$$

Since $u_{k}-u$ converges weakly to $u_{0}-u$ and $v_{k}-v$ converges strongly to $v_{0}-v$, we have

$$
\left(v_{k}-v, u_{k}-u\right) \rightarrow\left(v_{0}-v, u_{0}-u\right)
$$

Hence,

i.e.,

$$
\begin{gathered}
\left(v_{0}-v, u_{0}-u\right) \geqq 0, \\
\left(v_{0}-T(u), u_{0}-u\right) \geqq 0
\end{gathered}
$$

for all $u$ in $X$. By the maximal monotonicity of $T$, it follows that $v_{0} \in T\left(u_{0}\right)$. Q.E.D.

2. We begin the study of the ranges of monotone multi-valued mappings with the finite-dimensional case.

LEMMA 2.1. Let $F$ be a finite-dimensional Banach space, $F^{*}$ its conjugate space, $T$ a multi-valued mapping of $F$ into $F^{*}$. Suppose that $T$ is maximal 
monotone and that there exists a bounded subset $S$ of $F$ surrounding 0 such that for $u$ in $S$,

$$
(T(u), u) \geqq 0 .
$$

Then there exists $u_{0}$ in $K(S)$ such that $0 \in T\left(u_{0}\right)$.

Proof of Lemma 2.1. Since the hypotheses and conclusions are invariant under a change to an equivalent norm and since $F$ is of finite dimension, we may assume without loss of generality that $F$ is a Hilbert space and $F^{*}=F$.

We adopt a device used by Minty [15] under different hypotheses in infinitedimensional Hilbert spaces. For each positive integer $n$, let $T_{n}$ be the mapping from $X$ to $X^{*}$ whose graph is given by

$$
G\left(T_{n}\right)=\left\{\left[u+\frac{1}{n} v, v+\frac{1}{n} u\right] \mid[u, v] \in G(T)\right\} .
$$

We consider the properties of the mappings $T_{n}$. We begin by establishing the inequality:

$$
\left(w-w_{1}, x-x_{1}\right) \geqq \frac{1}{4 n}\left\{\left\|w-w_{1}\right\|^{2}+\left\|x-x_{1}\right\|^{2}\right\}
$$

for all $[x, w]$ and $\left[x_{1}, w_{1}\right]$ in $G\left(T_{n}\right)$. By the definition of $G\left(T_{n}\right)$, there exist $[u, v]$ and $\left[u_{1}, v_{1}\right]$ in $G(T)$ such that

$$
\begin{array}{ll}
x=u+\frac{1}{n} v, & w=v+\frac{1}{n} u, \\
x_{1}=u_{1}+\frac{1}{n} v_{1}, & w_{1}=v_{1}+\frac{1}{n} u_{1} .
\end{array}
$$

Hence,

$$
\begin{aligned}
\left(w-w_{1}, x-x_{1}\right) & =\left(\left(u-u_{1}\right)+\frac{1}{n}\left(v-v_{1}\right),\left(v-v_{1}\right)+\frac{1}{n}\left(u-u_{1}\right)\right) \\
& \geqq \frac{1}{n}\left\{\left\|u-u_{1}\right\|^{2}+\left\|v-v_{1}\right\|^{2}\right\}
\end{aligned}
$$

On the other hand,

$$
\begin{aligned}
& \left\|x-x_{1}\right\| \leqq\left\|u-u_{1}\right\|+\left\|v-v_{1}\right\|, \\
& \left\|w-w_{1}\right\| \leqq\left\|u-u_{1}\right\|+\left\|v-v_{1}\right\|
\end{aligned}
$$

so that

$$
\left\|x-x_{1}\right\|^{2}+\left\|w-w_{1}\right\|^{2} \leqq 4\left\{\left\|u-u_{1}\right\|^{2}+\left\|v-v_{1}\right\|^{2}\right\}
$$

and 


$$
\left(w-w_{1}, x-x_{1}\right) \geqq \frac{1}{4 n}\left\{\left\|x-x_{1}\right\|^{2}+\left\|w-w_{1}\right\|^{2}\right\} .
$$

As a corollary of the inequality (2.1), we see that if $x=x_{1}$, then $w=w_{1}$ and conversely so that $T_{n}$ is a one-to-one mapping with

$$
\frac{1}{4 n}\left\|x-x_{1}\right\| \leqq\left\|T_{n} x-T_{n} x_{1}\right\| \leqq 4 n\left\|x-x_{1}\right\| .
$$

If $T$ is maximal monotone, the transformation $T^{\#}$ with graph

$$
G\left(T^{\#}\right)=\left\{\left[u, \frac{v}{n}\right] \mid[u, v] \in G(T)\right\}
$$

is also maximal monotone. Applying Lemma 2 of Minty [13], we see that the set $\{u+v / n \mid[u, v] \in G(T)\}$ is the whole of $F$. Hence each $T_{n}$ is defined on all of $X$ and satisfies the inequality

$$
\left(T_{n} x-T_{n} x_{1}, x-x_{1}\right) \geqq \frac{1}{4 n}\left\|x-x_{1}\right\|^{2} .
$$

Hence by [13], each $T_{n}$ maps $F$ one-to-one onto $F$.

For each $n$, let $x_{n}$ be the unique solution of $T_{n} x_{n}=0$. Choose $\left[u_{n}, v_{n}\right] \in G(T)$ such that

$$
\begin{aligned}
& u_{n}+\frac{1}{n} v_{n}=x_{n}, \\
& v_{n}+\frac{1}{n} u_{n}=0 .
\end{aligned}
$$

We assert that $u_{n} \in K(S)$. Indeed for $u$ not in $K(S)$, we have $u=\rho u_{0}$, where $\rho>1, u_{0} \in S$ (since $S$ surrounds the origin). Since

$$
\left(T(u)-T\left(u_{0}\right), u-u_{0}\right) \geqq 0
$$

we have for $v \in T\left(u_{0}\right)$,

$$
\frac{(\rho-1)}{\rho}(T(u), u) \geqq(\rho-1)\left(T u_{0}, u_{0}\right) \geqq 0,
$$

i.e., for $v \in T(u),(v, u) \geqq 0$. For such $u$ and $v$

$$
\begin{aligned}
& \left(v+\frac{1}{n} u, v\right) \geqq\|v\|^{2}, \\
& \left(v+\frac{1}{n} u, u\right) \geqq \frac{1}{n}\|u\|^{2}
\end{aligned}
$$

so that if $v+(1 / n) u=0$, we have $u=0, v=0$, i.e., $u \in K(S)$, which is a contradictiof. Hence all the elements $u_{n}$ lie in $K(S)$. 
Since $K(S)$ is bounded, there exists a constant $M$ such that $\left\|u_{n}\right\| \leqq M$ for all $n$. Hence

$$
\left\|v_{n}\right\|=\left\|\frac{1}{n} u_{n}\right\| \leqq \frac{M}{n}
$$

so that $v_{n} \rightarrow 0$ as $n \rightarrow \infty$. We may choose a subsequence $\left\{u_{n_{j}}\right\}$ so that $u_{n j} \rightarrow u_{0}$ in $F$ as $j \rightarrow+\infty$. By Lemma 2.1, however, it follows that $0 \in T\left(u_{0}\right)$. Q.E.D.

LeMma 2.2. Let $T$ be a multi-valued mapping of $X$ into $X^{*}$ such that

(a) $T$ is monotone.

(b) $T$ is vaguely continuous.

(c) $T(u)$ is a bounded closed convex set for each $u$.

Let $Y$ be a closed subspace of $X$ such that $Y \subset D(T)$. Let $j$ be the injection mapping of $Y$ into $X, j^{*}$ the projection map of $X^{*}$ onto $Y^{*}$. Let $T_{1}$ be the multivalued mapping of $Y$ into $Y^{*}$ given by $T_{1}(u)=j^{*} T(j u)$ for $u$ in $Y$.

Then $T_{1}$ is monotone, $D\left(T_{1}\right)=Y$, and $T_{1}$ satisfies conditions (a), (b), and (c). In particular, $T_{1}$ is maximal monotone.

Proof of Lemma 2.2. For each $u$ in $Y, T(u) \neq \varnothing$ implies that $T_{1}(u) \neq \varnothing$. Hence $D\left(T_{1}\right)=Y$.

For $u, u_{1}$ in $Y$

$$
\left(T_{1}(u)-T_{1}\left(u_{1}\right), u-u_{1}\right)=\left(T(u)-T(u), u-u_{1}\right) \geqq 0
$$

so that $T_{1}$ is monotone.

Since $j^{*}$ is weakly continuous, if $v_{k} \in T\left(u_{k}\right)$ and $v_{k} \rightarrow v_{1}$ weakly in $X^{*}$ for $v_{1} \in T\left(u_{0}\right)$, then $j^{*} v_{k} \in T_{1}\left(u_{k}\right), j^{*} v_{1} \in T_{1}\left(u_{0}\right)$, and $j^{*} v_{k} \rightarrow j^{*} v_{1}$ weakly in $Y^{*}$. Hence $T_{1}$ is vaguely continuous.

Since $j^{*}$ is linear and $T(u)$ is convex for each $u, j^{*} T(u)=T_{1}(u)$ is convex for each $u$ in $Y$. Since $T(u)$ is a bounded closed convex set in the reflexive space $X^{*}$, it is weakly compact. Since $j^{*}$ is weakly continuous, $j^{*} T(u)=T_{1}(u)$ is weakly compact and hence closed. Thus we have completed the verification of properties (a), (b), and (c) for the mapping $T_{1}$.

Finally the maximal monotonicity of $T_{1}$ follows from (a), (b), and (c) and Theorem 1.2. Q.E.D.

THEOREM 2.1. Let $T$ be a multi-valued mapping of $X$ into $X^{*}$ such that $T(u)$ is bounded for each $u, D(T)$ is a linear subset of $X$, and for each closed line segment $S_{0}$ in $D(T)$, there exists a bounded set $S_{1}$ in $X^{*}$ (possibly depending on $S_{0}$ ) such that $T(u) \cap S_{1} \neq \varnothing$ for $u \in S_{0}$. Suppose further that

(i) $T$ is maximal monotone.

(ii) There exists a bounded subset $S$ of $X$ surrounding 0 such that

for $u \in S$.

$$
(T(u), u) \geqq 0
$$


Then there exists $u_{0}$ in $K(S)$ such that $0 \in T\left(u_{0}\right)$.

Proof of Theorem 2.1. Since $T$ is maximal monotone and a bounded set $S_{1}$ exists for each closed line segment $S_{0}$ such that $T(u) \cap S_{1} \neq \varnothing$ for $u \in S_{0}$, it follows from Theorem 1.1 that $T$ is vaguely continuous, and $T(u)$ is a bounded closed convex subset of $X^{*}$ for each $u$ in $D(T)$.

Let $F$ be a finite-dimensional subspace of $D(T)$. Let $j_{F}$ be the injection mapping of $F$ into $X, j_{F}^{*}$ the dual map projecting $X^{*}$ onto $F^{*}$. We form the mapping $T_{F}: F \rightarrow F^{*}$ by setting $T_{F} u=j_{F}^{*}\left(T_{F}\left(j_{F} u\right)\right)(u \in F)$. Then by Lemma $2.2, T_{F}$ is vaguely continuous, $T_{F}(u)$ is a closed convex subset of $F^{*}$ for every $u$ in $F, D\left(T_{F}\right)=F$, and $T_{F}$ is a monotone multi-valued mapping of $F$ into $F^{*}$. Hence by Theorem 1.2, $T_{F}$ is a maximal monotone mapping of $F$ into $F^{*}$.

Let $S_{F}=S \cap F$. Then $S_{F} \subset K\left(S_{F}\right) \subset K(S)$, and $S_{F}$ surrounds the origin in $F$. For $u$ in $S_{F}$,

$$
\left(T_{F}(u), u\right)=\left(j_{F}^{*} T(u), u\right)=(T(u), u) \geqq 0 .
$$

Hence $T_{F}$ satisfies the hypotheses of Lemma 2.1 and there exists $u_{F}$ in $K\left(S_{F}\right) \subset K(S) \cap F$ such that $0 \in T_{F}\left(u_{F}\right)$.

For any $u$ in $F$, we have, however,

i.e.,

$$
\left(T_{F}\left(u_{F}\right)-T_{F}(u), u_{F}-u\right) \geqq 0,
$$

$$
\left(T(u), u-u_{F}\right) \geqq 0
$$

Hence, the set

$$
V_{F}=\{v \mid v \in K(S),(T(u), u-v) \geqq 0\} \text { for all } u \in F
$$

is a nonempty weakly closed convex subset of the weakly compact set $K(S)$ in $X$. Since the family of such sets is closed under finite intersections, it follows that the set

$$
\bigcap_{F} V_{F} \neq \varnothing
$$

If $u_{0}$ lies in $\bigcap_{F} V_{F}$, however, $u_{0}$ lies in $K(S)$, and

$$
\left(T(u), u-u_{0}\right) \geqq 0
$$

for all $u \in D(T)$. Hence by the maximal monotonicity of $T, 0 \in T\left(u_{0}\right)$. Q.E.D.

THEOREM 2.2. Let $T$ be a multi-valued mapping of $X$ into $X^{*}$ such that $D(T)=X, T$ is monotone and vaguely continuous, and $T(u)$ is a bounded closed convex set for each $u$. Suppose that there exists a bounded set $S$ surrounding 0 in $X$ such that $(T(u), u) \geqq 0$ for $u$ in $S$.

Then there exists $u_{0}$ in $K(S)$ such that $0 \in T\left(u_{0}\right)$. 
Proof of Theorem 2.2. This is the same as that of Theorem 2.1 except that the vague continuity of $T$ is given to us by hypothesis and does not need to be deduced from maximal monotonicity and the existence of sets $S_{1}$ as in Theorem 2.1.

THEOREM 2.3. Let $T$ be a monotone multi-valued mapping of $X$ into $X^{*}$ $Y$ a closed subspace of $X, Y^{\perp}$ its annihilator in $X^{*}$. Suppose that $Y \subset D(T)$ and that there exists a subset $S$ surrounding 0 in $Y$ such that $(T(u), u) \geqq 0$ for $u$ in S. Suppose also that one of the two following conditions holds:

(A) $T$ is maximal monotone. $T(u)$ is a bounded set for each $u$, and for each closed segnent $S_{0}$ in $X$, there exists a bounded set $S_{1}$ in $X^{*}$ such that $T(u) \cap S_{1} \neq \varnothing$.

(B) $T$ is vaguely continuous and $T(u)$ is a bounded closed convex subset of $X^{*}$ for each $u$.

Then there exists $u_{0}$ in $K(S) \subset Y$ such that $T\left(u_{0}\right) \cap Y^{\perp} \neq \varnothing$.

Proof of Theorem 2.3. If $j$ is the injection mapping of $Y$ into $X, j^{*}$ the projection mapping of $X^{*}$ on $Y^{*}$, we set $T_{1}(u)=j^{*}(T(u))$. Then $T\left(u_{0}\right) \cap Y^{\perp} \neq \varnothing$ if and only if $0 \in T_{1}(u)$. If (A) holds, $T_{1}$ satisfies the hypotheses of Theorem 2.1, while if (B) holds, $T_{1}$ satisfies the hypotheses of Theorem 2.2. Hence our conclusion follows. Q.E.D.

THEOREM 2.4. Let $T$ be a monotone multi-valued mapping of $X$ into $X^{*}$, $Y$ a closed subspace of $X$ with $Y \subset D(T), Y^{\perp}$ the annihilator of $Y$ in $X^{*}$. Suppose that $T$ satisfies either of the conditions (A) and (B) of Theorem 2.3 and that there exists a continuous real-valued function on $R^{1}$ with $c(r) \rightarrow+\infty$ as $r \rightarrow+\infty$ such that

for $u \in Y$.

$$
(T(u), u) \geqq c(\|u\|)\{\|u\|+\|T(u)\|\}
$$

Then for each $v_{0}$ in $X, w_{0}$ in $X^{*}$,

$$
T\left(Y+v_{0}\right) \cap\left(w_{0}+Y^{\perp}\right) \neq \varnothing .
$$

Proof of Theorem 2.4. We form the mapping $T^{\#}$ of $X$ into $X^{*}$ by setting

$$
T^{\#}(u)=T\left(u_{0}+v_{0}\right)-w_{0} .
$$

Then $T^{\#}$ satisfies the hypotheses of Theorem 2.3 with respect to $Y$ since for $\|u\|$ sufficiently large

$$
\begin{aligned}
\left(T\left(u+v_{0}\right)-w_{0}, u\right)= & \left(T\left(u+v_{0}\right), u+v_{0}\right)-\left(w_{0}, u\right)-\left(T\left(u+v_{0}\right), v_{0}\right) \\
\geqq & c\left(\left\|u+v_{0}\right\|\right)\left\{\left\|u+v_{0}\right\|+\left\|T\left(u+v_{0}\right)\right\|\right\}-\left\|w_{0}\right\| \cdot\|u\| \\
& -\left\|v_{0}\right\| \cdot\left\|T\left(u+v_{0}\right)\right\| \geqq 0 . \quad \text { Q.E.D. }
\end{aligned}
$$


It is interesting to compare Theorem 2.3 with the result obtained by Minty in [15]. In our notation, this is the following:

THEOREM (MinTy). Let $H$ be a Hilbert space, $T$ a multi-valued mapping of $H$ into $H, Y$ a closed subspace of $H$. Suppose that $T$ is maximal monotone and satisfies all of the following conditions:

(i) $(T(u), u) \geqq-c$ for some $c>0$ and all $u$ in $H$.

(ii) There exists a bounded set $C$ surrounding 0 in $H$ such that for every $u$ in $C$, there exists $v \in T(u)$ such that

$$
(v, u) \geqq 0 \text {. }
$$

(iii) There exists a bounded set $D$ in $H$ surrounding 0 such that for each $v \in D$, there exists $u$ in $H$ such that $v \in T(u)$ and

$$
(v, u) \geqq 0 \text {. }
$$

Then $T(X) \cap Y^{\perp} \neq \varnothing$.

To clarify the relation of this result to Theorem 2.3, we note that by the monotonicity of $T$, the condition (ii) of Minty's theorem is equivalent to the stronger condition:

(ii)' $C \subset D(T)$ and $(T u), u) \geqq 0$ for $u \in C$.

Indeed if $k>1$ is fixed and $u \in C$, we have from condition (ii):

$$
0 \leqq(T(k u)-v, k u-u)=(k-1)\left\{\frac{1}{k}(T(k u), k u)-(v, u)\right\} .
$$

Hence if $u_{1}=k u \in k C,\left(T\left(u_{1}\right), u_{1}\right) \geqq 0$.

Theorem 2.4 is thus a generalization of Minty's theorem to reflexive Banach spaces with hypotheses (i) and (iii) dropped and with the additional hypotheses that $T(u)$ is bounded for each $u$ and that for each line segment $S_{0}$, there exists a bounded set $S_{1}$ intersecting $T(u)$ for all $u$ in $S_{0}$,

3. Let $X$ be a reflexive Banach space as before, $X^{*}$ its conjugate space, $\phi$ a continuous nondecreasing non-negative function of $r$ in $R^{1}$ with $\phi(0)=0$, $\phi(r) \rightarrow+\infty$ as $r \rightarrow+\infty$.

Definition. If $u \neq 0$ is an element of $X, v$ in $X^{*}$ is said to be a dual element to $u$ with respect to the gauge function $\phi$ if

$$
\begin{aligned}
& (v, u)=\|v\| \cdot\|u\|, \\
& \|v\|=\phi(\|u\|) .
\end{aligned}
$$

Definition. The duality map $T$ of $X$ into $X^{*}$ (with respect to the gauge function $\phi)$ is given by $T(0)=0$ and for $u \neq 0$,

$$
T(u)=\{v \mid v \text { is dual to } u\} \text {. }
$$


LEMMA 3.1. If $X$ is a reflexive Banach space, $\phi$ a continuous non-negative nondecreasing function on $R^{1}$ with $\phi(0)=0$, then the duality map $T$ of $X$ into $X^{*}$ with respect to $\phi$ is a multi-valued maximal monotone mapping of $X$ into $X^{*}$ with $D(T)=X$ and

(a) $T$ is vaguely continuous.

(b) $T(u)$ is a bounded closed convex subset of $X^{*}$ for each $u$ in $X$.

(c) For all $u$ in $X$

where

$$
(T(u), u) \geqq c(\|u\|)\{\|u\|+\|T u\|\},
$$

$$
c(r)=\min \left\{\frac{1}{2} r, \frac{1}{2} \phi(r)\right\} .
$$

Proof of Lemma 3.1. The maximal monotonicity of $T$ will follow if we prove that $T$ is monotone, $D(T)=X$, and (a), (b), and (c) above are valid. $D(T)=X$ by the Hahn-Banach theorem. If $u, u_{1} \in X$ and $v \in T(u), v_{1} \in T\left(u_{1}\right)$, then

$$
\begin{aligned}
\left(v-v_{1}, u-u_{1}\right) & =\|v\| \cdot\|u\|+\left\|v_{1}\right\| \cdot\left\|u_{1}\right\|-\left(v, u_{1}\right)-\left(v_{1}, u\right) \\
& \geqq\|v\| \cdot\|u\|+\left\|v_{1}\right\| \cdot\left\|u_{1}\right\|-\|v\| \cdot\left\|u_{1}\right\|-\left\|v_{1}\right\| \cdot\|u\| \\
& =\left(\|v\|-\left\|v_{1}\right\|\right)\left(\|u\|-\left\|u_{1}\right\|\right) \\
& =\left(\phi(\|u\|)-\phi\left(\left\|u_{1}\right\|\right)\right)\left(\|u\|-\left\|u_{1}\right\|\right) \geqq 0,
\end{aligned}
$$

since $\phi$ is nondecreasing. Hence $T$ is monotone.

Proof of (a). Let $\left\{u_{k}\right\}$ be a sequence converging strongly to $u_{0}, v_{k} \in T\left(u_{k}\right)$. Then $\left\|v_{k}\right\|=\phi\left(\left\|u_{k}\right\|\right) \leqq M$, so that by extracting a subsequence, we can assume that $v_{\dot{k}} \rightarrow v_{1}$ weakly in $X^{*}$. Since $u_{k} \rightarrow u_{0}$ strongly, we have

while

$$
\left\|v_{k}\right\| \cdot\left\|u_{k}\right\|=\left(v_{k}, u_{k}\right) \rightarrow\left(v_{1}, u_{0}\right)
$$

Hence

$$
\begin{aligned}
\left\|v_{1}\right\| & \leqq \liminf \left\|v_{k}\right\|, \\
\left\|u_{0}\right\| & =\lim \left\|u_{k}\right\| .
\end{aligned}
$$

$$
\left\|v_{1}\right\| \cdot\left\|u_{0}\right\| \leqq\left(v_{1}, u_{0}\right) \leqq\left\|v_{1}\right\| \cdot\left\|u_{0}\right\| \cdot
$$

Thus

$$
\left(v_{1}, u_{0}\right)=\left\|v_{1}\right\| \cdot\left\|u_{0}\right\|
$$

Moreover

so that

$$
\left(v_{1}, u_{0}\right)=\lim \left(v_{k}, u_{k}\right)=\lim \phi\left(\left\|u_{k}\right\|\right)\left\|u_{k}\right\|=\phi\left(\left\|u_{0}\right\|\right)\left\|u_{0}\right\|
$$

$$
\left\|v_{1}\right\|=\phi\left(\left\|u_{0}\right\|\right)
$$

Thus $v_{1} \in T\left(u_{0}\right)$. 
Proof of (b). Obviously $T(u)$ is bounded and closed. Suppose $v, v_{1} \in T(u)$ Then for $0 \leqq t \leqq 1$,

$$
\begin{aligned}
\left(t v+(1-t) v_{1}, u\right) & =t(v, u)+(1-t)\left(v_{1}, u\right) \\
& =t \phi(\|u\|)\|u\|+(1-t) \phi(\|u\|)\|u\| \\
& =\phi(\|u\|)\|u\|
\end{aligned}
$$

However, if $v_{t}=t v+(1-t) v_{1}$, we have

$$
\left\|v_{t}\right\| \leqq t\|v\|+(1-t)\left\|v_{1}\right\|=\phi(\|u\|) .
$$

Hence

$$
\left(v_{t}, u\right)=\phi(\|u\|)\|u\| \geqq\left\|v_{t}\right\|\|u\|
$$

and since

$$
\left(v_{t}, u\right) \leqq\left\|v_{t}\right\| \cdot\|u\|,
$$

we have $\left\|v_{t}\right\|=\phi(\|u\|)$ and $v_{t} \in T(u)$. Hence $T(u)$ is convex. Q.E.D.

Proof of (c). For $u \in X$

$$
\begin{aligned}
(T u, u) & =\phi(\|u\|)\|u\|=\frac{1}{2}\|T(u)\| \cdot\|u\|+\frac{1}{2} \phi(\|u\|)\|u\| \\
& \geqq c(\|u\|)\{\|u\|+\|T(u)\|\} . \quad \text { Q.E.D. }
\end{aligned}
$$

THEOREM 3.1. Let $X$ be a reflexive Banach space, $Y$ a closed subspace of $X, X^{*}$ the conjugate space of $X, Y^{\perp}$ the annihilator of $Y$ in $X^{*}$. Let Tbe a duality map of $X$ into $X^{*}$. If $v_{0} \in X, w_{0} \in X^{*}$, then the set

$$
T\left(Y+v_{0}\right) \cap\left(Y^{\perp}+w_{0}\right) \neq \varnothing .
$$

Proof of Theorem 3.1. By Lemma 3.1, $T$ satisfies the hypotheses of Theorem 2.4 and our conclusion follows.

Q.E.D.

\section{BIBLIOGRAPHY}

1. A. Beurling and A.E. Livingston, $A$ theorem on duality mappings in Banach spaces, Ark Mat. 4 (1962), 405-411. $557-566$

2. F. E. Browder, Solvability of non-linear functional equations, Duke Math. J. 30 (1963),

3. - Variational boundary value problems for quasi-linear elliptic equations of arbitrary order, Proc. Nat. Acad. Sci. U.S.A. 50 (1963), 31-37.

4. - Variational boundary value problems for quasi-linear elliptic equations. II, Proc. Nat. Acad. Sci. U.S.A. 50 (1963), 592-598.

5. _- Variational boundary value problems for quasi-linear elliptic equations. III, Proc. Nat. Acad. Sci. U.S.A. 50 (1963), 794-798.

6. —_ Non-linear elliptic boundary value problems, Bull. Amer. Math. Soc. 69 (1963), 862-874. 
7. - Non-linear parabolic boundary value problems of arbitrary order, Bull. Amer. Math. Soc. 69 (1963), 858-861.

8. - Strongly non-linear parabolic boundary value problems, Amer. J. Math. 86 (1964), 339-357.

9. - Nonlinear elliptic boundary problems, II Trans. Amer. Math. Soc. 117 (1965), $530-550$.

10. - Nonlinear elliptic problems. II, Bull. Amer. Math. Soc. 70 (1964), 299-302.

11. - Non-linear equations of evolution Ann. of Math. 80 (1964), 485-523.

12. - On a theorem of Beurling and Livingston, Canad. J. Math. 17 (1965).

13. G. J. Minty, Monotone (non-linear) operators in Hilbert space, Duke Math. J. 29 (1962), 341-346.

14. - On a "monotonicity" method for the solution of non-linear equations in Banach spaces, Proc. Nat. Acad. Sci. U.S.A. 50 (1963), 1038-1041.

15. - Unpublished paper on maximal monotone sets for Hilbert spaces.

\author{
INSTITUTE FOR ADVANCED STUDY, \\ Princeton, NeW Jersey \\ UNIVERSITY OF CHICAGo, \\ Chicago, Illinois
}

